\title{
COMPARISON OF TWO FEATURE EXTRACTION METHODS BASED ON MAXIMIZATION OF MUTUAL INFORMATION
}

\author{
Nikolay Chumerin, Marc M. Van Hulle \\ K.U.Leuven, Laboratorium voor Neuro- en Psychofysiologie \\ Campus Gasthuisberg, Herestraat 49, B-3000 Leuven, BELGIUM \\ E-mail: nick.chumerin@student.kuleuven.be,marc@neuro.kuleuven.be
}

\begin{abstract}
We perform a detailed comparison of two feature extraction methods that are based on mutual information maximization between the data points projected in the developed subspace and their class labels. For the simulations, we use synthetic as well as publicly available real-world data sets.
\end{abstract}

\section{INTRODUCTION}

Dimensionality reduction is a widespread preprocessing step in high-dimensional data analysis, visualization and modeling. One of the simplest ways to reduce dimensionality is by Feature Selection (FS): one selects only those input dimensions that contain the relevant information for solving the particular problem. Feature Extraction (FE) is a more general method in which one tries to develop a transformation of the input space onto the low-dimensional subspace that preserves most of the relevant information. We will further focus on linear FE methods which means that they can be represented by a linear transformation $\mathbf{W}: \mathbb{R}^{D} \rightarrow \mathbb{R}^{d}$, $D>d$. Feature Extraction methods can be supervised or unsupervised, depending on whether or not class labels are used. Among the unsupervised methods, Principal Component Analysis (PCA) [1], Independent Component Analysis (ICA) [2], and Multidimensional Scaling (MDS) [3] are the most popular ones. Supervised FE methods (and also FS methods) either use information about the current classification performance, called wrappers, or use some other, indirect measure, called filters. One expects that, in the case of a classification problem, supervised methods will perform better than unsupervised ones.

Recently, a method has been introduced by Torkkola [4] that has attracted a lot of attention. Consider the data set

\footnotetext{
NC is supported by the Belgian Fund for Scientific Research - Flanders (G.0248.03)

MMVH is supported by the Excellence Financing program of the K.U.Leuven (EF 2005), the Belgian Fund for Scientific Research - Flanders (G.0248.03, G.0234.04), the Flemish Regional Ministry of Education (Belgium) (GOA 2000/11), the Belgian Science Policy (IUAP P5/04), and the European Commission (NEST-2003-012963, IST-2002-016276, IST2004-027017))
}

$\left\{\mathbf{x}_{i}, c_{i}\right\}, i=1, \ldots, N$ with $\mathbf{x}_{i} \in \mathbb{R}^{D}$ the data points, and $c_{i}$ the class labels taken from the discrete set $\mathcal{C}=\left\{c_{p}\right\}, p=$ $1, \ldots, N_{c}$. The objective is to find a linear transformation $\mathbf{W} \in \mathbb{R}^{D \times d}$ for which the mutual information (MI) of the transformed data points $Y=\left\{\mathbf{y}_{i}\right\}=\left\{\mathbf{W}^{T} \mathbf{x}_{i}\right\}$ and the corresponding labels $C=\left\{c_{i}\right\}$ is maximized. The objective is different from ICA's where MI between the transformed data components is minimized. Also, the presence of the labels $C$ makes the objective different. Torkkola derived an expression for MI based on Renyi's quadratic entropy [5], instead of Shannon's entropy, and a plug-in density estimate based on Parzen windowing.

Prior to Torkkola, Bollacker and Ghosh [6] proposed an incremental approach to MI maximization that was derived by rewriting the original MI objective function as a sum of MI terms between the one-dimensional projections and the corresponding class labels. A polytope algorithm was used for the optimization and histograms for estimating the probabilities. Very recently, a method based on the same reformulation of the MI objective function was introduced by Leiva-Murillo and Artés-Rodríguez (2006) [7]. However, they used gradient descent as an optimization strategy, and expressed the one-dimensional MI terms as onedimensional negentropies, which were then estimated using Hyvärinen's robust estimator [8].

The purpose of this paper is to perform an in-depth comparison of the two MI based FE methods. The paper is structured as follows. Section 2 briefly describes the FE method based on quadratic MI maximization, as proposed by Torkkola. In Section 3 we also briefly describe the approach proposed by Leiva-Murillo and Artés-Rodríguez. In Section 4, we describe a number of measures for FE comparison, and in Section 5 we explain our comparison methodology. The results of the comparison are given in Section 6, followed by a Discussion in Section 7.

\section{TORKKOLA'S METHOD}

Given two random variables $X_{1}$ and $X_{2}$ with joint probability density $p\left(x_{1}, x_{2}\right)$ and marginal probability densities 
$p_{1}\left(x_{1}\right)$ and $p_{2}\left(x_{2}\right)$, the mutual information (MI) can be expressed as:

$$
I\left(X_{1}, X_{2}\right)=K\left(p\left(x_{1}, x_{2}\right), p_{1}\left(x_{1}\right) p_{2}\left(x_{2}\right)\right),
$$

with $K(\cdot, \cdot)$ the Kullback-Leibler divergence.

In order to estimate MI, Torkkola and Campbell [4] use the quadratic measures $K_{C}$ or $K_{T}$ originally introduced by Principe and co-workers [5]:

$$
\begin{aligned}
K_{C}(f, g) & =\log \frac{\int f^{2}(\mathbf{x}) d \mathbf{x} \int g^{2}(\mathbf{x}) d \mathbf{x}}{\left(\int f(\mathbf{x}) g(\mathbf{x}) d \mathbf{x}\right)^{2}} \\
K_{T}(f, g) & =\int(f(\mathbf{x})-g(\mathbf{x}))^{2} d \mathbf{x} .
\end{aligned}
$$

For continuous-valued $Y$ and discrete-valued $C$, using (1), (2) and (3), one can derive two types of MI estimates:

$$
\begin{aligned}
I_{C}(Y, C) & =\log \frac{V_{(c y)^{2}} V_{c^{2} y^{2}}}{\left(V_{c y}\right)^{2}}, \\
I_{T}(Y, C) & =V_{(c y)^{2}}+V_{c^{2} y^{2}}-2 V_{c y},
\end{aligned}
$$

where:

$$
\begin{aligned}
V_{(c y)^{2}} & =\sum_{c \in \mathcal{C}} \int_{\mathbf{y}} p^{2}(\mathbf{y}, c) d \mathbf{y} \\
V_{c^{2} y^{2}} & =\sum_{c \in \mathcal{C}} \int_{\mathbf{y}} p^{2}(c) p^{2}(\mathbf{y}) d \mathbf{y} \\
V_{c y} & =\sum_{c \in \mathcal{C}} \int_{\mathbf{y}} p(\mathbf{y}, c) p(c) p(\mathbf{y}) d \mathbf{y} .
\end{aligned}
$$

The class probability can be evaluated as $p\left(c_{p}\right)=J_{p} / N$, where $J_{p}$ is the number of samples in class $c_{p}$. The density of the projected data $p(\mathbf{y})$ and the joint density $p(\mathbf{y}, c)$ are estimated with the Parzen window approach [9]:

$$
\begin{aligned}
p(\mathbf{y}) & =\frac{1}{N} \sum_{i=1}^{N} G\left(\mathbf{y}-\mathbf{y}_{i}, \sigma^{2} I\right) \\
p\left(\mathbf{y}, c_{p}\right) & =\frac{1}{N} \sum_{i=1}^{J_{p}} G\left(\mathbf{y}-\mathbf{y}_{p j}, \sigma^{2} I\right),
\end{aligned}
$$

with $G(\mathbf{x}, \Sigma)$ the Gaussian kernel with center $\mathbf{x}$ and covariance matrix $\Sigma$, and $\mathbf{y}_{j p}$ the $j$-th sample in class $c_{p}$. In order to reduce the number of parameters to optimize, Torkkola proposes a parametrization of the desired matrix $\mathbf{W}$ in terms of Givens rotations in $\mathbb{R}^{D}$. As a result, there are only $d(D-d)$ parameters (rotation angles) to optimize instead of $D^{2}$. Obviously, the maximal number of parameters to estimate occurs for $d$ near $D / 2$. The computational complexity of the method is claimed to be $O\left(N^{2}\right)$.

\section{ARTÉS-RODRÍGUEZ METHOD}

In the Artés-Rodríguez method, an objective function (global MI) in terms of the sum of individual MI's is considered:

$$
I_{A R}(Y, C)=\sum_{i=1}^{d} I\left(y_{i}, c\right)=\sum_{i=1}^{d} I\left(\mathbf{w}_{i}^{T} \mathbf{x}, c\right),
$$

with $y_{i}=\mathbf{w}_{i}^{T} \mathbf{x}$ the data projected onto direction $\mathbf{w}_{i}$, and $\mathbf{w}_{i} \in \mathbb{R}^{D}$ the $i$-th column of the desired orthonormal matrix W.

Assuming the original data is whitened, each individual MI can be estimated as:

$$
I\left(y_{i}, c\right)=\sum_{p=1}^{N_{c}} p\left(c_{p}\right)\left(J\left(y_{i} \mid c_{p}\right)-\log \sigma\left(y_{i} \mid c_{p}\right)\right)-J\left(y_{i}\right),
$$

with $y_{i} \mid \mathrm{c}_{p}$ the projection of the $p$-th class' data points onto the $\mathbf{w}_{i}$ direction, $J(\cdot)$ the negentropy, and $\sigma(\cdot)$ the standard deviation. Hyvärinen's robust estimator [8] for the negentropy is used:

$$
\begin{aligned}
J(z) \approx & k_{1}\left(E\left\{z \exp \left(-z^{2} / 2\right)\right\}\right)^{2} \\
& +k_{2}\left(E\left\{\exp \left(-z^{2} / 2\right)\right\}-\sqrt{1 / 2}\right)^{2},
\end{aligned}
$$

with $k_{1}=36 /(8 \sqrt{3}-9)$ and $k_{2}=24 /(16 \sqrt{3}-27)$. In a top-down scheme, one should sequentially (thus, one-byone) obtain the projection directions $\mathbf{w}_{i}$ thereby preserving the two constraints: $\left\|\mathbf{w}_{i}\right\|=1$ and $\mathbf{w}_{i}^{T} \mathbf{w}_{j}=0$ for $1 \leq j<i$. The second constraint means that each projection direction must be searched in the subspace orthogonal to the projection directions already obtained, and this causes the search for each new projection direction to be carried out in a subspace of decreasing dimension. The sequence of individual MI's obtained in this way is also decreasing: $I\left(y_{i}, c\right)>I\left(y_{j}, c\right)$ for $i<j$. The bottom-down scheme involves a sequential removing of the directions with minimum individual MI's between the variables and classes.

\section{MEASURES FOR COMPARISON}

In order to compare the two FE methods, we use four different MI estimators: the two mentioned above, $I_{C}$ and $I_{A R}$, the binned estimator $I_{B}$, and the one proposed by Kraskov and co-workers [10], namely, the $I^{(2)}$ estimator (rectangular version).

The most straightforward, and most widely used method to estimate the MI between two variables $X$ and $Y$ is the histogram-based approach. The support of each variable is partitioned into bins of finite size. Denoting by $n_{x}(i)$ $\left(n_{y}(j)\right)$ the number of points falling in $i$-th bin of $X(j$-th 
bin of $Y)$, and $n(i, j)$ the number of points in their intersection, we can estimate MI:

$$
I_{B}(X, Y)=\log N+\frac{1}{N} \sum_{i, j} n(i, j) \log \frac{n(i, j)}{n_{x}(i) n_{y}(j)}
$$

Unfortunately this estimator is biased, even in the case of adaptive partitioning. Another disadvantage of the binned estimator is the high memory requirements in the high-dimensional case.

Kraskov MI estimator $I^{(2)}$ is based on entropy estimation using $k$-nearest neighbour statistics. Let $X$ and $Y$ are normed spaces with norms $\|\cdot\|_{X}$ and $\|\cdot\|_{Y}$ respectively. Consider new space $Z=X \times Y$ with norm $\|\cdot\|_{Z}$ which for every $\mathbf{z} \in Z, \mathbf{z}=(\mathbf{x}, \mathbf{y})$ is defined as

$$
\|\mathbf{z}\|_{Z}=\max \left\{\|\mathbf{x}\|_{X},\|\mathbf{y}\|_{Y}\right\} .
$$

For fixed natural $k$ let us denote by $\epsilon(i) / 2$ the distance from $\mathbf{z}_{i}$ to its $k$-th neighbour, and by $\epsilon_{x}(i) / 2$ and $\epsilon_{y}(i) / 2$ the distances between the same points projected into the $X$ and $Y$ subspaces. Denoting by

$$
\begin{aligned}
& n_{x}(i)=\#\left\{\mathbf{x}_{j}:\left\|\mathbf{x}_{i}-\mathbf{x}_{j}\right\|_{X} \leq \epsilon_{x}(i) / 2\right\} \\
& n_{y}(i)=\#\left\{\mathbf{y}_{j}:\left\|\mathbf{y}_{i}-\mathbf{y}_{j}\right\|_{Y} \leq \epsilon_{y}(i) / 2\right\}
\end{aligned}
$$

MI can be estimated by

$$
I^{(2)}(X, Y)=\psi(k)-\frac{1}{k}-\left\langle\psi\left(n_{x}\right)+\psi\left(n_{y}\right)\right\rangle+\psi(N),
$$

here $\langle\ldots\rangle=N^{-1} \sum_{i=1}^{N} E\{\ldots(i)\}$ is averaging operation both over all $i=1, \ldots, N$ and over all realizations of the random samples and $\psi(x)$ is digamma function:

$$
\psi(x)=\frac{1}{\Gamma(x)} \frac{d \Gamma(x)}{d x} .
$$

It satisfies the recursion $\psi(x+1)=\psi(x)+1 / x$ and $\psi(1)=$ $-C$ where $C=0.5772156 \ldots$ is the Euler-Mascheroni constant. For large $x, \psi(x) \approx \log x-1 / 2 x$.

\section{COMPARISON METHODOLOGY}

In order to have a fair comparison, we use the original source code of the Artés-Rodríguez algorithm (courtesy of LeivaMurillo and Artés-Rodríguez) and the publicly available implementation of Torkkola's approach MeRMaId-SIG by Kenneth E. Hild II [11]. For the Artés-Rodríguez algorithm, we choose the top-down scheme. We consider both synthetic and real world data sets. The synthetic data set consists of a variable number of equal-sized, normally distributed clusters (modes) in $\mathbb{R}^{D}$. The clusters centers are Gaussianly distributed with variance equal to 3 . All data sets are centered and whitened before applying the respective

\begin{tabular}{llll}
\hline Data set name & $\begin{array}{l}\text { Dimension Number } \\
(D)\end{array}$ & $\begin{array}{l}\text { Number } \\
\text { of samples } \\
(N)\end{array}$ & $\begin{array}{l}\text { of classes } \\
\left(N_{c}\right)\end{array}$ \\
\hline Iris & 4 & 150 & 3 \\
Pima & 8 & 500 & 2 \\
Glass & 9 & 214 & 7 \\
Pipeline flow & 12 & 1000 & 3 \\
Wine & 13 & 178 & 3 \\
\hline
\end{tabular}

Table 1. Information about used real data sets

FE methods. We consider $N_{c}=3, \ldots, 10$ clusters, and use 1000 data sets with $d=1, \ldots, D-1$ subspace dimensions. The MI estimators' means and standard deviations for the 1000 data sets are then plotted as a function of the subspace dimensionalities $d$. For the real-world data sets, we compute the MI estimates for each possible subspace dimension $d$. The Pipeline Flow data set was taken from Aston University ${ }^{1}$. The rest of the real-world data sets were taken from the UCI Machine Learning Repository ${ }^{2}$. If the data dimensionality was more than 9 , we did not evaluate $I_{B}$ (binned estimator) due to memory limitations.

The algorithms are implemented using quite different, yet simple optimization techniques: the Artés-Rodríguez algorithm employs a simple adaptation of the learning rate during evaluation, while the MeRMaId-SIG uses a pure gradient ascent with constant learning rate and fixed number of iterations. Due to this, a fair comparison of the run times is not straightforward. Therefore, we determine the number of float-point operations (flops) needed for one gradient evaluation of each algorithm. It is a more relevant measure than the average computing time because it does not depend on the optimization techniques used by these algorithms.

The flops were obtained using the flops function (in Matlab 5.3) on data sets with $N \in\{1000,2000,3000,4000\}$ and $D \in\{4,8,12,16\}$.

\section{RESULTS}

For the synthetic data sets we show only the case of $D=6$, $N=1000$ and $N_{c}=5$ (Figs. 1-4). The results for the real-world data sets are shown in Figs. 5-8 and in Table 2.

The speed comparison results are shown in Table 3 . The case $D=8$ for Torkkola's method is shown in Fig. 9 in more detail. We do not show the plots for the Artés-Rodríguez approach because each gradient evaluation needs the same number of flops for all $d=1, \ldots, D-1$.

For data sets with fixed numbers of samples $N$, fixed dimensions $D$ and different numbers of clusters $N_{c}$, gradient evaluation in both methods needs almost the same numbers of floating point operations (the deviation in flops for

\footnotetext{
${ }^{1}$ http://www.ncrg.aston.ac.uk/GTM/3PhaseData.html

${ }^{2}$ http://www.ics.uci.edu/ mlearn
} 


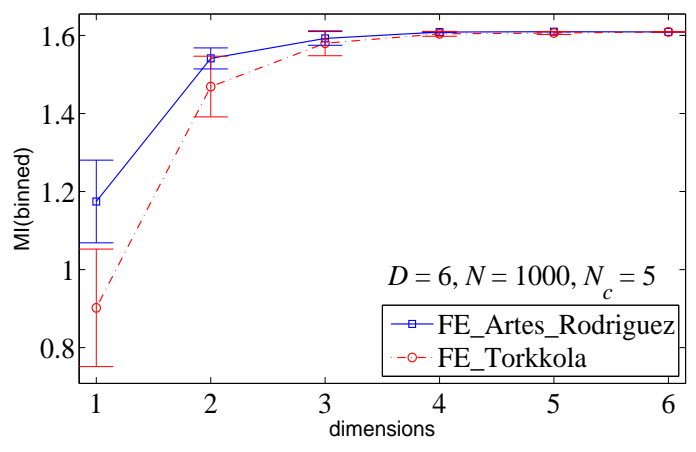

Fig. 1. Mean of $I_{B}$ vs. $d$

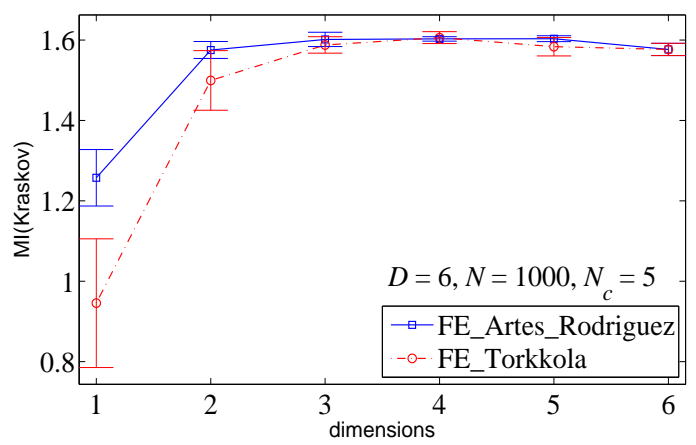

Fig. 2. Mean of $I^{(2)}$ vs. $d$

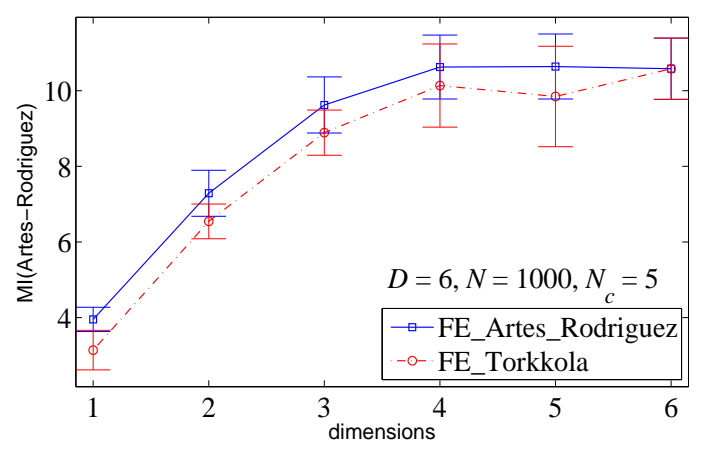

Fig. 3. Mean of $I_{A R}$ vs. $d$

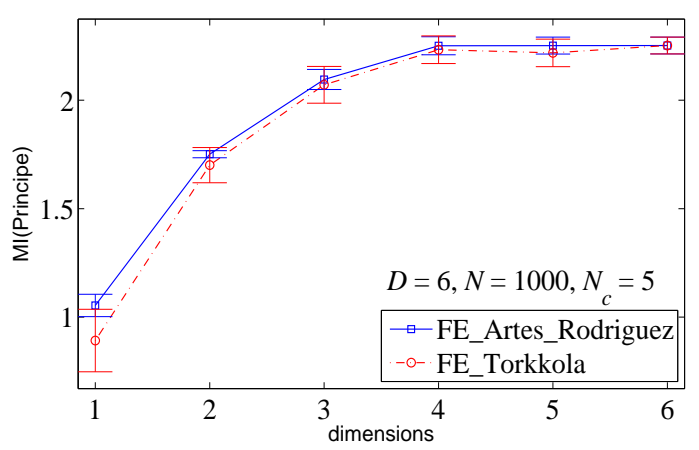

Fig. 4. Mean of $I_{C}$ vs. $d$

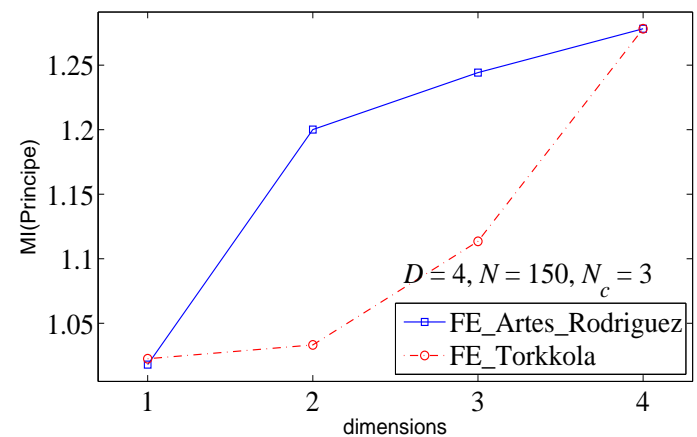

Fig. 5. $I_{C}$ versus $d$ for Iris Plants Database

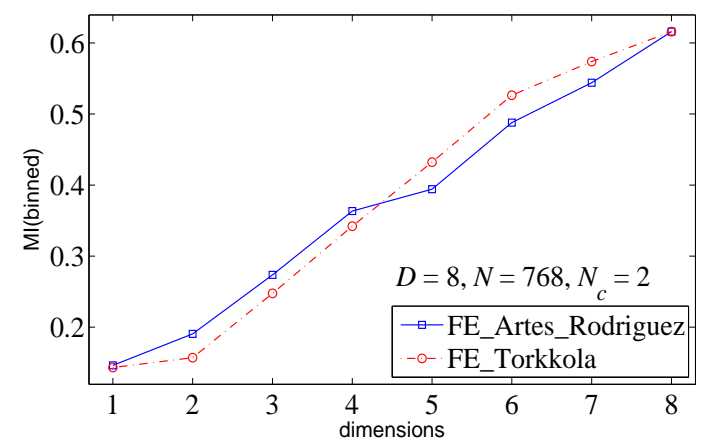

Fig. 6. $I_{B}$ versus $d$ for Pima Indians Diabetes Database

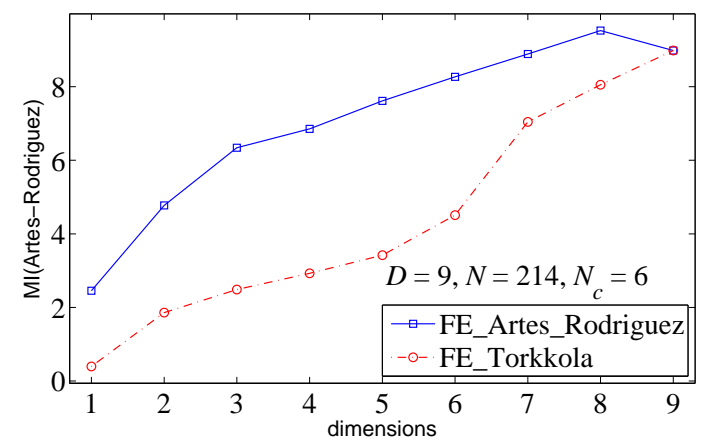

Fig. 7. $I_{A R}$ versus $d$ for Glass Identification Database

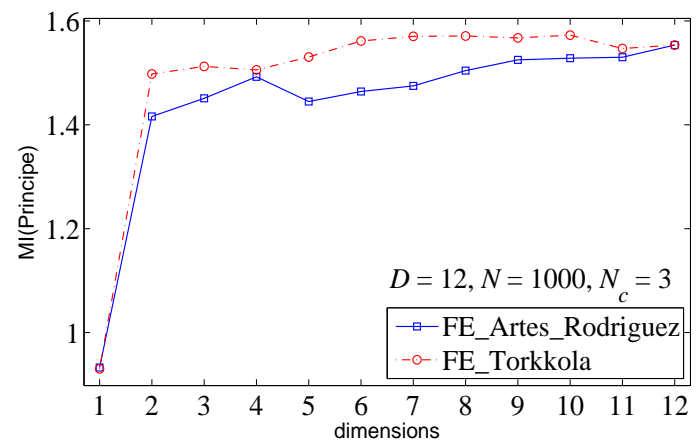

Fig. 8. $I_{C}$ versus $d$ for Pipeline Flow data 


\begin{tabular}{llcccc}
\hline Data set & Approach & $\left\langle I_{B}\right\rangle$ & $\left\langle I_{C}\right\rangle$ & $\left\langle I_{A R}\right\rangle$ & $\left\langle I^{(2)}\right\rangle$ \\
\hline Iris & AR & 1.0391 & 1.1541 & 5.0251 & 0.9944 \\
& Torrkola & 1.0181 & 1.0565 & 4.0409 & 0.9561 \\
\hline Pima & AR & 0.3428 & 0.2089 & 0.8528 & 0.1628 \\
& Torrkola & 0.3461 & 0.2026 & 0.4140 & 0.1678 \\
\hline Glass & AR & 0.7078 & 0.4212 & 6.8401 & 0.5952 \\
& Torrkola & 0.7430 & 0.4409 & 3.8368 & 0.4764 \\
\hline Pipeline & AR & 1.0814 & 1.4331 & 20.461 & 1.0668 \\
& Torrkola & 1.0749 & 1.4889 & 5.9973 & 1.0605 \\
\hline \multirow{2}{*}{ Wine } & AR & 1.0668 & 1.5019 & 8.3007 & 0.8798 \\
& Torrkola & 0.9009 & 1.2422 & 3.0588 & 0.7194 \\
\hline
\end{tabular}

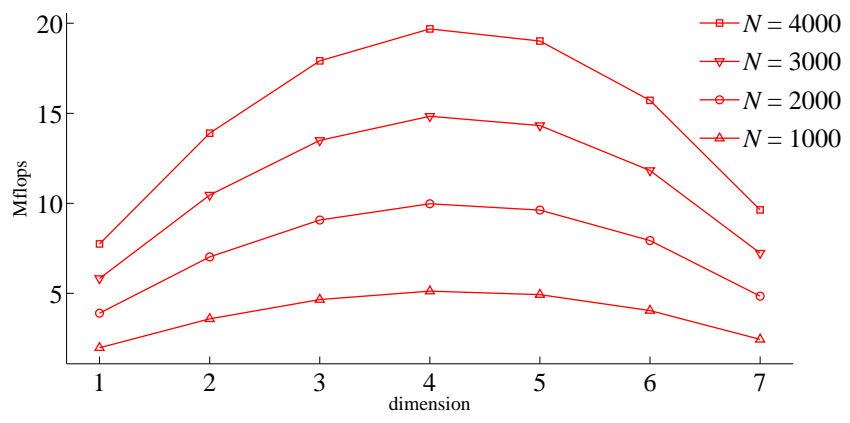

Table 2. Averages of the estimated MI for all real data sets considered and $d=1, \ldots, D-1 ;\left\langle I_{B}\right\rangle$. Note that the estimates were computed only for $d<9$ ) (see text).

\begin{tabular}{cccc}
\hline$D$ & $N$ & Torkkola & Artés-Rodríguez \\
\hline 4 & 1000 & 0.165 & $0.253 \ldots 0.390$ \\
& 2000 & 0.329 & $0.505 \ldots 0.778$ \\
& 3000 & 0.493 & $0.757 \ldots 1.166$ \\
& 4000 & 0.657 & $1.009 \ldots 1.554$ \\
\hline 8 & 1000 & 0.438 & $1.976 \ldots 5.121$ \\
& 2000 & 0.874 & $3.900 \ldots 9.977$ \\
& 3000 & 1.310 & $5.824 \ldots 14.833$ \\
& 4000 & 1.746 & $7.748 \ldots 19.689$ \\
\hline 12 & 1000 & 0.841 & $6.977 \ldots 27.540$ \\
& 2000 & 1.677 & $13.517 \ldots 50.544$ \\
& 3000 & 2.513 & $20.057 \ldots 73.548$ \\
& 4000 & 3.349 & $26.597 \ldots 96.552$ \\
\hline 16 & 1000 & 1.372 & $17.556 \ldots 104.446$ \\
& 2000 & 2.736 & $33.192 \ldots 175.022$ \\
& 3000 & 4.100 & $48.828 \ldots 245.598$ \\
& 4000 & 5.464 & $64.464 \ldots 316.174$ \\
\hline
\end{tabular}

Fig. 9. Plots of the floating point operations required for Torkkola's gradient evaluation for $D=8$ and different $N$. It should not come as a surprise that the shape of plots reflect the quadratic nature of the number of parameters to optimize (see text).

Table 3. Comparison of floating point operations (in Mflops) needed for one gradient evaluation.

constant $N, D$ and $N_{c} \in\{5,10,20\}$ was less than $1 \%$ ). This is the reason why we present here the comparison only for $N_{c}=5$ and different $N$ and $D$ values. The CPU time should grow with increasing $N_{c}$, however, it stays almost constant. We explain this by the highly optimized manner Matlab treats matrix computations: for fixed $N$, the more classes we have, the more portions of the data (with smaller sizes) are processed in a vectorized manner.

\section{DISCUSSION}

The results show that, for most data sets, the Artés-Rodríguez approach yields better results. From our point of view, one of the reasons of the better performance of the ArtésRodríguez algorithm is the fact that $I_{A R}$ is more smoother and has less local optima than the other measures, includ-

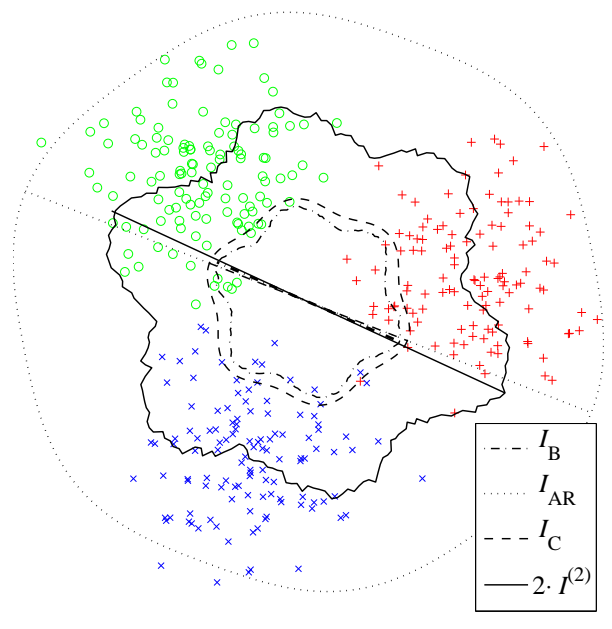

Fig. 10. Plots of $I_{B}, I_{A R}, I_{C}$ and $I^{(2)}$ (which is doubled for the sake of exposition) as a function of the angle of the direction on which the data points are projected, given a two-dimensional data set consisting of 3 equal sized Gaussian clusters. For each plot the direction of the maxima is indicated with a line segment. It can be clearly seen that $I_{A R}$ is more smoother than the other measures, with almost coinciding maxima. 
ing the $I_{C}$ metric used in Torkkola's, with almost coinciding maxima. This is illustrated in Fig. 10. One should also remind that in the Artés-Rodríguez approach, for all computations of the gradient, one-dimensional projections are used, whereas Torkkola's approach gradient evaluations are based on data of dimensionality $d(D-d)$. This could be beneficial as well.

Another issue is data preprocessing. In Torkkola's only PCA is used as data preprocessing, whereas Artés-Rodríguez employs a more sophisticated technique: successively PCA and SIR (Sliced Inverse Regression) are used, which already yields a quite good MI result.

One should also mention that due to the better optimization technique the Artés-Rodríguez algorithm is much faster than MeRMaId-SIG.

In summary, the Artés-Rodríguez approach is not only robust but also fast and reliable, and promises a successful future.

\section{REFERENCES}

[1] J.E. Jackson, A User's Guide to Principal Components, Wiley, New York, 1991.

[2] A. Hyvärinen, J. Karhunen, and E. Oja, Independent component analysis, John Wiley \& Sons, 2001.

[3] F.W. Young, "Multidimensional scaling: History, theory, and applications," R.M. Hamer, Ed. 1987, Hillsdale, NJ: Lawrence Erlbaum Associates.

[4] K. Torkkola and W. Campbell, "Mutual information in learning feature transformations.," in ICML, 2000, pp. 1015-1022.

[5] J.C. Principe, J.W. Fisher III, and D. Xu, "Information theoretic learning," in Unsupervised Adaptive Filtering, Simon Haykin, Ed., New York, 2000, Wiley.

[6] K.D. Bollacker and J. Ghosh, "Linear feature extractors based on mutual information," in Proceedings of the 13th International Conference on Pattern Recognition, 1996, vol. 2, pp. 720-724.

[7] A. Artés-Rodríguez and J. M. Leiva-Murillo, "Maximization of mutual information for supervised linear feature extraction," submitted.

[8] A. Hyvärinen, "New approximations of differential entropy for independent component analysis and projection pursuit," in Advances in Neural Information Processing Systems, Michael I. Jordan, Michael J. Kearns, and Sara A. Solla, Eds. 1998, vol. 10, pp. 273279, The MIT Press.
[9] E. Parzen, "On the estimation of a probability density function and mode," Annals of Mathematical Statistics, vol. 33, pp. 1065-1076, 1962.

[10] A. Kraskov, H. Stögbauer, and P. Grassberger, "Estimating mutual information," 2003.

[11] K.E. Hild II, D. Erdogmus, K. Torkkola, and J.C. Principe, "Sequential feature extraction using information-theoretic learning," in press. 